ARTICLE

\title{
Development of a scale of Chinese primary school teachers' job embeddedness
}

\author{
Xuguang Sun (1) ${ }^{1 凶} \&$ Ailing Huang ${ }^{1}$
}

\begin{abstract}
Based on the background of Chinese education reform and Chinese cultural values, the paper aims to develop a good reliable and valid scale to measure the job embeddedness of Chinese primary school teachers. To achieve the above purpose, first, we created these domains and items through an analysis of previous literature and an opened questionnaire survey of Chinese primary school teachers. Second, three experts and scholars from education and psychology evaluated the content validity of the scale. Finally, we randomly selected 534 Chinese primary school teachers to form a sample, and then tested the reliability and validity of the scale by SPSS and Amos software. We found that the scale of Chinese primary school teachers' job embeddedness consists of five domains, including 28 items. The reliability and validity of the scale is acceptable. The five domains were named "fit to organization", "fit to community", "organization-related sacrifice", "links to community" and "fit to family". Therefore, we come to the following conclusions: first, the structural model of job embeddedness of Chinese primary school teachers consists of five dimensions. Second, the scale gets a good reliability and validity.
\end{abstract}

\footnotetext{
${ }^{1}$ School of Education, Fujian Normal University, Fujian, China. ${ }^{凶}$ email: 752191721@qq.com
} 


\section{Introduction}

conomic historian Karl Polanyi first proposed the concept of "embeddedness", which refers to "the human economy is embedded and entangled in economic and non-economic institutions." In 1985, Granovetter further elaborated "embeddedness" proposed by Karl Polanyi. He believes that economic behavior is embedded in the social structure, and the core of the social structure is the social network in people's lives. Social members differentially occupy and allocate scarce resources according to connection. The theory fields generally believe that the concept of "embeddedness" has a certain universality, not only applicable to the economic field, but also to other human social activities. The embedded can be mortal; the embedded person is the economic and cultural structure of society. In this way, the concept of embeddedness is pretty flexible, so that the concept of "embeddedness" has the capacity of transfer. Not only are economic activities embedded in social relationships, but people, as social actors, are also in a certain social structure or embedded in the social structure.

Based on the above theoretical development, Mitchell et al. (2001) brought embeddedness into the field of work, proposing the concept of job embeddedness. Mitchell et al. (2001) believe that job embeddedness is how much individuals depend on organizations or surroundings, including three elements: fit, link, sacrifice. Specifically, fit refers to whether the employee's work and community are consistent with their values, goals and plans; Links are a formal or informal relationship established between employees and organizations; Sacrifice refers to the loss suffered by employees after resigning and leaving the residential community. Since then, job embeddedness has quickly become a research hotspot, and many researchers have first studied from the concept and its theoretical construction.

In foreign studies, almost all studies support job embeddedness theory proposed by Mitchell et al. (2001), which divides job embeddedness into two aspects: organization and community. For example, Lee et al. (2004) believe that the structure of job embeddedness is composed of organization and community, and community is off-the-job embeddedness, organization is on-thejob embeddedness. Burton et al. (2010) found that on-the-job embeddedness can effectively decrease the negative influence on organizational citizenship behavior and job performance.

While proposing the concept of "job embeddedness", Mitchell et al. (2001) conducted a survey of employees in enterprises and hospitals and compiled a scale of job embeddedness. The scale was revised according to the needs of the sample, and Lee et al. (2004) divided job embeddedness into two parts: on-the-job embeddedness and off-the-job embeddedness. Crossley et al. (2007) developed and tested a comprehensive measuring tool of job embeddedness, which overcomes the weakness of synthetic measuring job embeddedness.

People constantly change and develop in connection with the experience we have as individual, human beings in a specific cultural and social context (Bondebjerg, 2017). In cross-cultural research, the Chinese not only give attention to organization and community, but also pay more attention to family factor. Chinese scholars selected 16 employees from different industries who had previous resignation experience and found that the influence of family and relatives of employees' turnover intention is particularly prominent (Yang et al. 2013). Zhong (2015) compared with the West, Chinese employees pay more attention to family-related factors, rather than factors related to community, proposing the concept of "family embeddedness" to explain employee turnover. Song (2016) believes that the job embeddedness in the Chinese context should include links to organization, fit to organization, organization-related sacrifice, interpersonal relationships, and fit to environment and family-related sacrifice.
In Chinese traditional culture, the concept that "family harmony symbolizes prosperity" is emphasized. At the same time, under the influence of collective culture, collectivists usually live near their parents and even close friends. Therefore, for Chinese, family is of great importance. Chinese not only value the impact of organizations and communities on work, but also the influence of family on work. This makes the structural model of primary school teachers' job embeddedness different from the West. Teachers are part of society and naturally are affected by various factors. Primary schools always recruit teachers, but primary school teachers often are turnover, called "revolving doors". It is shown that primary school teachers have a higher turnover. And job embeddedness is the latest theory to explain turnover. Therefore, job embeddedness can be applied in the field of education to explain the problems of education human resource management. However, on the one hand, by searching Chinese and foreign literature databases, we find that only few researchers have studied teachers' job embeddedness. For example, in foreign studies, some researchers found that on-the-job embeddedness plays a mediating role between conscientiousness and teachers' contextual performance (Lev. and Koslowsky, 2012). Rajappan et al. (2017) found that middle school teachers in Ernakulam were well embedded, and the conclusion also explains the positive correlativity between the spirit of workplace and job embeddedness. In China, Zhang (2011) studied the structure of high school teachers' job embeddedness and developed a questionnaire of Chinese high school teachers' job embeddedness.

On the other hand, we find that although researchers have conducted cross-cultural research on job embeddedness, they have only used qualitative methods to research or developed research tools that not effectively combine with the actual Chinese context. Therefore, based on Chinese cultural values and existing research on job embeddedness, this paper will develop a scale of job embeddedness of primary school teachers.

\section{Purpose}

Study 1. Study 1 aims to explore the structure of Chinese primary school teachers' job embeddedness and form a pre-test questionnaire. First, we conducted to search the existing literature of job embeddedness. Second, through an opened survey of Chinese primary school teachers' job embeddedness, we tried to develop the initial items.

Study 2. Study 2 aims to establish the content structure of the job embeddedness of primary school teachers and form the formal scale.

\section{Method}

We assume that the structural model of the primary school teachers' job embeddedness is a second-order factor construction model. First, in the section "Study 1: pre-test questionnaire", exploratory factor analysis (EFA) was carried out to extract common factors to form first-order factors. Second, in the section "Study 2: formal questionnaire", we performed a confirmatory factor analysis (CFA) to establish the relationship between the first-order factors and the measurement variables (measurement variables are items on the scale) and the relationship between first-order factors. We tested the fitting index to attain the purpose. In addition, we made convergence validity and discriminant validity to effectively reflect the validity of the structural model. For convergence validity, Kline (2011) believed that the judgment of convergence validity should be consistent with the following characteristics: each observation index has a relatively high normalized factor load. The direction is consistent, and the correlation between factors is not too high. For discriminant validity, 
$\mathrm{Wu}$ (2013) pointed out that the average variance extraction (AVE) of each dimension is greater than the square of the correlation coefficient of each dimension, indicating that the discriminant validity of the scale is good.

\section{Study 1: pre-test questionnaire}

Participants. When preparing the initial questionnaire, we interviewed 10 front-line teachers. When preparing the pre-test questionnaire, we selected 180 participants. Among them, valid questionnaires were 150 , and the rate of effective recovery was $93.2 \%$.

Method. Firstly, we searched the existing literature by major educational research databases (including CNKI, Web of Science, and Google Scholar) to learn the research status. To reduce the risks of bias, we included all relevant publications in our analysis, including qualitative and quantitative research, peer-reviewed journals, systematic reviews, books, etc.

Second, in the process of questionnaire preparation, the formation of questionnaire items is of great importance. Only after consulting a large amount of literature and in-depth analysis, combined with sufficient interviews, can we truly identify the job embedded questionnaire items that reflect the characteristics of the primary school teachers. 4 open-ended questions of primary school teachers' job embeddedness were investigated to meet the Chinese cultural context: (a) What factors do you think will keep you working in school? (b) Do you consider that the backbone teacher training has an effect on your stay in school? (c) How would you rate your relationship with the school, family and residential community? (d) What factors do you believe can improve your work embedding?

Third, before factor analysis, we first checked the scale whether is suitable for factor analysis. If the KMO value is less than 0.5 . It is not appropriate to carry out factor analysis (Kasier, 1974). Then, eigenvalue greater than 1 were extracted by the Kaiser's standardized optimal skew method, one of the principal component analysis method, and scree plot (Kasier, 1974). Finally, the items with the commonality greater than 0.20 and the factor loading greater than 0.45 are retained. At the same time, further adjust the items with vague and ambiguous meanings.

Finally, after the first three steps, we discussed with experts and deleted some items, forming an initial questionnaire. After the last step, we formed a pre-test questionnaire.

\section{Study 2: formal questionnaire}

Participants. In total, 534 participants that job embeddedness of Chinese primary school teachers were from a random sample, including 154 male and 380 female Chinese primary school teachers $\left(M_{\text {age }}=1.76, \mathrm{SD}=0.80\right)$. Randomly selected about half of the participants (Sample $1=266, N_{\text {male }}=73, N_{\text {female }} 193, M_{\text {age }}=$ $1.76, \mathrm{SD}=0.81)$ used SPSS 23.0 for exploratory factor analysis (EFA). The remaining 268 participants (Sample $2=268, N_{\text {male }}=$ $\left.81, N_{\text {female }}=187, M_{\text {age }}=1.76, \mathrm{SD}=0.79\right)$ were selected for confirmatory factor analysis (CFA). It is worth noting that all participants in the pre-test and formal investigations volunteered to participate in the survey with their consent. The study was conducted in strict accordance with the requirements of ethical standards and was supervised by psychologists and pedagogical experts.

Instrument and data collection. The formal scale of Chinese primary school teachers' job embeddedness consists of five domains, includes 28 items, using a Likert five-point scoring format. And basic demographic information such as gender, age, and teaching age was collected. The scale was completed by Chinese primary school teachers from seven provinces in eastern China via electronic and paper questionnaires.

Data analyses. The statistical analyses divided into four steps. We conducted a missing value check before performing data analysis. First, an exploratory factor analysis (EFA) was used to determine the common factors. We used principal component extraction, the eigenvalue $(>1)$ and the scree plot, determining the number of common factors. A promax rotation was used according to the advice by Meyers et al. (2017). Next, we screened items according to following standards: (a) Factor's eigenvalue is more than 1; (b) Each item's factor loadings should be more than 0.45; (c) Every factor covers more than 3 items; (d) Factor loading is unique; (e) Factors can be easier to name (Zhang and Lu, 2017).

Second, CFA was performed using Amos 21.0 to check the validity of the scale to verify the validity of the aforementioned five-domain structure. Specific steps are as follows (Wu, 2013): Drawing a CFA model $\rightarrow$ Selecting SPSS data $\rightarrow$ Selecting measurement variables and defining first-order factors $\rightarrow$ Setting model estimation method and performing model estimation $\rightarrow$ Determining whether the measurement model is fitting with the data. According to Hair et al. (2010), fit indices including Chi-square degree of freedom $\left(\chi^{2} / d f\right)$, root-mean-square error of approximation (RMSEA), the comparative fit index (CFI), Tucker-Lewis index (TLI), and incremental fit index (IFI) were examined to test the overall model fit to the data. $1<\chi^{2} / d f<3$, RMSEA $<0.08$, CFI $>0.90$, TLI $>0.90$, IFI $>0.90$ have been recommended as a reference standard for a fit model (Bagozzi and Yi, 1988).

Third, we calculated the mean number, standard deviation and correlation coefficient of the primary school teachers' job embeddedness questionnaires and indicated that the five factors are a connected overall structure.

Finally, after removing items, we calculated cronbach's alpha using SPSS 23.0 according to Cohen's criteria (Cohen et al., 2013) to examine the internal consistency of the items and the entire scale and further tested convergence validity and discriminant validity to effectively reflect the validity of the structural model. Convergence validity means that the indicators that measure the same trait will be about the same factor. At the same time, the indicator will be under a higher factor loading in this factor structure. It suggests that the construct has good validity. Hair et al. (2010) believe that the convergence validity can be tested from the following aspects: (a) the factor loadings (or standardized path coefficient) are higher than 0.50; (b) the average variance extraction (AVE) is greater than 0.50 ; (c) the composite reliability reaches 0.6 at least. In addition to assessing whether there is a good convergence efficiency, it should also be evaluated whether there is good discriminant validity between different factors. If a multi-factor model lacks discriminant validity, it means that multiple factor constructs can synthesize a single factor. Wu (2013) believes that the average variance extraction (AVE) of each factor should be more than the square of the correlation coefficient between factors.

\section{Results}

Result: study 1. After data analysis, the KMO value here is 0.89 , which means that there are common factors between the items and the scale is suitable to make factor analysis. $\chi^{2}$ value is 3294.40 by Bartlett's spherical test, which is suitable to make the factor analysis. The pre-test questionnaire was determined to consist of 28 items.

Result: study 2. First, we used Sample 1 to conduct an exploratory factor analysis (EFA) of the formal questionnaire. After testing, the sample's KMO value was 0.92 , and $\chi^{2}$ value is 5313.92 
Table 1 Reliability and EFA results of the job embeddedness of Chinese primary school teachers.

\begin{tabular}{lll} 
Factor & Cronbach's $\boldsymbol{\alpha}$ & Eigenvalue \\
\hline Fit to organization & 0.95 & 11.87 \\
Fit to community & 0.90 & 2.75 \\
Organization-related sacrifice & 0.78 & 1.86 \\
Links to community & 0.83 & 1.44 \\
Fit to family & 0.77 & 1.10 \\
Entire scale & 0.95 & - \\
\hline
\end{tabular}

\begin{tabular}{|c|c|c|c|c|c|}
\hline & 1 & 2 & 3 & 4 & 5 \\
\hline Fit to organization & 1 & - & - & - & - \\
\hline Fit to community & $0.45^{\star \star}$ & 1 & - & - & - \\
\hline Organization-related sacrifice & $0.50^{\star \star}$ & $0.48^{\star \star}$ & 1 & - & - \\
\hline Links to community & $0.41^{\star \star}$ & $0.38^{\star \star}$ & $0.27^{\star \star}$ & 1 & - \\
\hline Fit to family & $0.65^{\star \star}$ & $0.39^{\star \star}$ & $0.46^{\star \star}$ & $0.30^{\star \star}$ & 1 \\
\hline
\end{tabular}

by Bartlett's spherical test, which can do factor analysis. Finally, items are no longer deleted, and formal questionnaire with 5 dimensions has 28 items. In total, five factors counted for $67.95 \%$ of the total interpretation rate of the entire scale. Based on the common characteristics of each item within its domains, the first factor was defined as "fit to organization", the second factor was defined as "fit to community", the third factor was defined as "organization-related sacrifice", the fourth factor was defined as "links to community", and the other factor was defined as "fit to family". The results of EFA are given in Table 1.

Second, CFA results show that the model has a good fit index $\left(\chi^{2} / d f=2.22 ; \quad\right.$ RMESA $=0.07 ; \quad$ TLI $=0.91 ; \quad$ CFI $=0.92$; IFI $=0.92)$.

Third, correlation analyses showed that there is a moderate correlation between items, $r=0.27-0.65$. Correlation analyses results are shown in Table 2. What's more, the results show that the correlation between the primary school teachers' job embeddedness in each item range from 0.57 to 0.89 (see Table 3 ), which is moderate and high correlation. It indicates that five factors constitute an ideal structural model of primary school teachers' job embeddedness.

Finally, the reliability of each factor is $0.95,0.90,0.78,0.83$, 0.77 , respectly. The reliability of the entire scale is 0.95 (see Table 1). According to the above criteria, the factor loadings of 28 items are higher than 0.50 , which can effectively reflect the factor characteristics; The average variance extractions (AVE) of the five factors are $0.58,0.65,0.54,0.64$, and 0.56 , respectively, which are higher than the critical value of 0.50 , suggesting that the model has good convergence efficiency; The composite reliability of the five factors are $0.95,0.90,0.78,0.84$, and 0.79 , both of which are more than 0.70 , indicating that the model has good internal quality. In addition, the result shows that Chinese primary school teachers' job embeddedness in the CFA model has good discriminant validity, and there are significant differences in each factor (see Table 4).

\section{Discussion}

It is the first attempt to develop a scale of Chinese primary school teachers' job embeddedness. According to a large amount of evidence, family is a necessary factor in teachers' job embeddedness (Zhong, 2015) and in practice (Yang et al., 2013), we believe that the scale can provide an important reference for future research on teachers' job embeddedness.

Scale domains and item content. Five domains of Chinese primary school teachers' job embeddedness were identified in this study:

Fit to organization refers to the degree of fit between the culture, values, and goals of primary school teachers and the school in which they are employed, and the identification of leadership. According to the theory of Person-Organization Fit by Edwaeds et al. (2006), primary school teachers are attracted to schools that match their values. Conversely, primary school teachers have a tendency to resign when they feel unfit for school culture, values and so on.

Fit to community is defined as a teacher's perceived compatibility with community. Enjoyable atmosphere of the community and the comfortable living environment can promote teachers' development physically and mentally. In this sense, the level of job embeddedness will improve. On the contrary, without basic sense of security or inconvenient transportation, primary school teachers will inevitably be affected.

Organization-related sacrifice reflects the cost of what people go to have to give up if they leave a job. Zhang (2002) believes that teachers' careers are closely linked to family life. Liu (2018) is convinced that family and life are of great significance to teachers. For example, the effect on teachers from the potential conflicts between work, family and life even exceeds the effect of personal career development goals.

Links to community means the degree of harmony between primary school teachers and residents living in the community, reflecting whether teachers' work is recognized and respected by residents. According to McClelland Achievement Motivation Theory (1961), people are anxious for a sense of belonging. Therefore, primary school teachers are expected to establish friendly and close interpersonal relationships and yearn for a high degree of mutual understanding.

Fit to family indicates teachers' compatibility to effectively handle relationships between home and work. Clark (2000) holds that individuals wander between work and family every day, coordinating the boundary between work and family, which affects the individual relationship in diverse fields. Particularly, family affects every aspect of individual social life in China. Therefore, whether work and family life can be balanced will affect the job embeddedness of primary school teachers.

Application value of the scale. We recommend using a fivedomain model to study the future job embeddedness of Chinese primary school teachers. However, before using this scale in other countries or other subject fields, it needs to be revalidated. For example, workplace spirit is a factor in the scale of middle school teachers' job embeddedness in Ernakulam area of India (Rajappan et al., 2017).

On the one hand, based on the entire scale score obtained, the scale can be used to recruit primary school teachers and assess the level of primary school teachers' job embeddedness. Zhang (2011), for example, revealed that these practices should be necessary in addition to knowledge and teaching skill test scores. In the process of enrolling primary school teachers, it is necessary to consider the degree of job embeddedness of primary school teachers. The score of primary school teachers' job embeddedness provides an useful suggestions for screening suitable primary school teachers. Therefore, the scale can help primary schools to reduce turnover and improve school teaching effectiveness.

On the other hand, using the scale of Chinese primary school teachers' job embeddedness, it is possible to actively improve the 


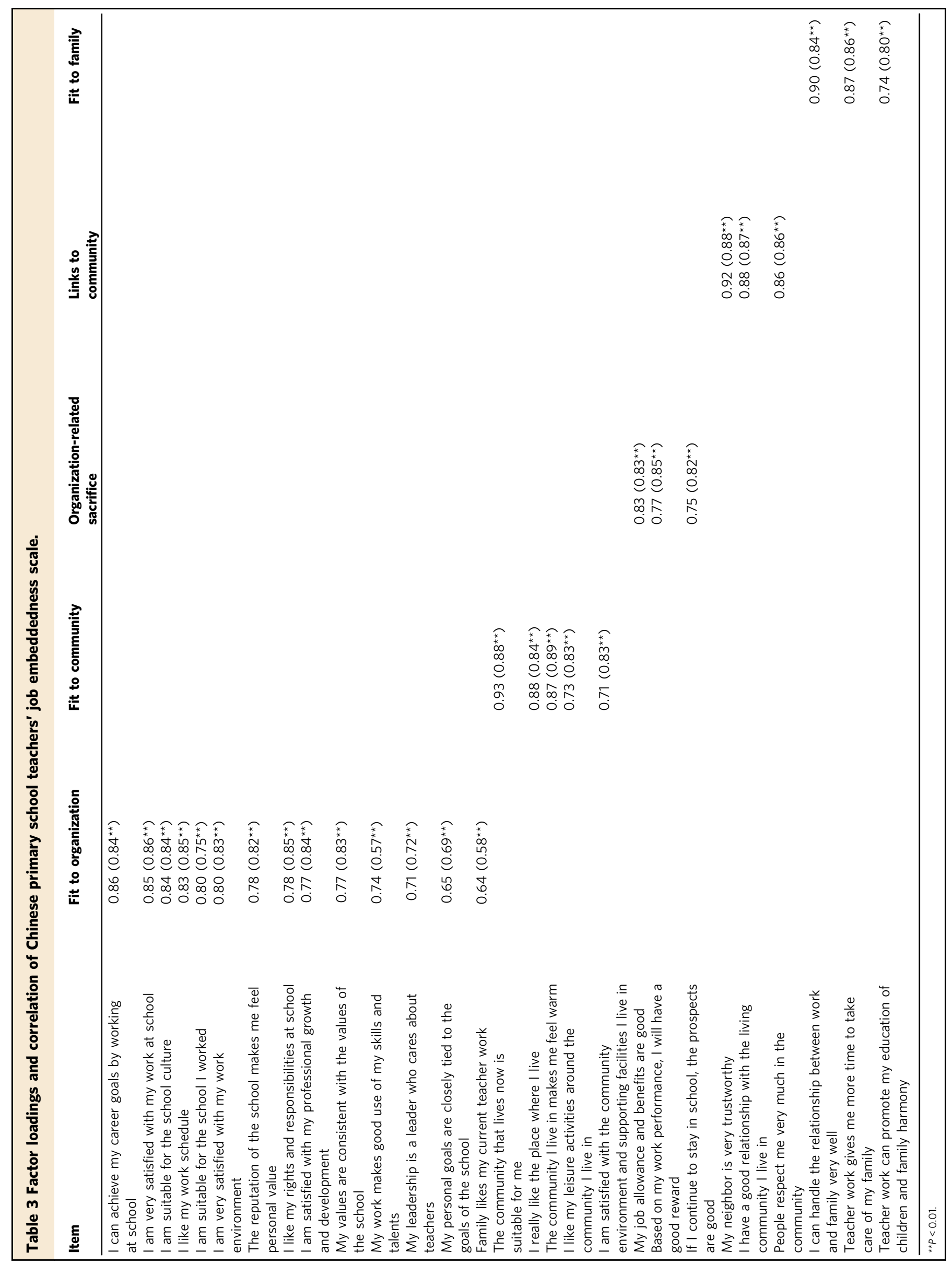


Table 4 The average variance extraction (AVE) of five factors.

\begin{tabular}{|c|c|c|c|c|c|}
\hline Factor & fit to organization & fit to community & $\begin{array}{l}\text { organization-related } \\
\text { sacrifice }\end{array}$ & links to community & fit to family \\
\hline Fit to organization & 0.58 (AVE) & $0.29(r 2)$ & $0.32(r 2)$ & $0.31(r 2)$ & $0.45(r 2)$ \\
\hline Fit to community & $0.54^{\star \star \star}$ & 0.65 (AVE) & $0.25(r 2)$ & $0.35(r 2)$ & $0.32(r 2)$ \\
\hline $\begin{array}{l}\text { Organization-related } \\
\text { sacrifice }\end{array}$ & $0.57^{\star \star \star}$ & $0.50^{\star \star \star}$ & 0.54 (AVE) & $0.28(r 2)$ & $0.37(r 2)$ \\
\hline Links to community & $0.56^{\star \star \star}$ & $0.59^{\star \star \star}$ & $0.53^{\star \star \star}$ & 0.64 (AVE) & $0.31(r 2)$ \\
\hline Fit to family & $0.67^{\star \star \star}$ & $0.57^{\star \star \star}$ & $0.61^{\star \star \star}$ & $0.56^{\star \star \star}$ & 0.56 (AVE) \\
\hline
\end{tabular}

Note: The diagonal is the factor constructing the mean variance extraction (AVE); the lower triangle is the correlation coefficient between factors, and the upper triangle is the square of the correlation coefficient between factors.

$\star \star \star P<0.001$

status of work of primary school teachers and provide some inspiration for the reform of education management in the new era.

\section{Conclusions}

The study offers an essential scale for measuring job embeddedness of Chinese primary school teachers in the Chinese population. Through the exploratory factor analysis and confirmatory factor analysis, the structural model of Chinese primary school teachers' job embeddedness consists of five dimensions: fit to organization, fit to community, organization-related sacrifice, links to community and fit to family, with good reliability and validity.

There are some limitations that are to be noted. First, this study is a scale developed in the context of Chinese culture. Chinese people focus on the impact of family on work. However, it may not apply in other countries. Therefore, this study only provides an effective example of the research on teachers' job embeddedness, provides inspiration for future researchers to study teachers' job embeddedness, telling researchers to conduct research in combination with the cultural background and the actual environment of the country. Mallol et al. (2007) and Young-bohk and Jeong-ran (2009) believe that cross-cultural research should pay attention to the characteristics of different cultural groups and adjust the original structure of job embeddedness. Only in this way can we provide practical management advice for solving the problem of turnover in diverse cultural groups. Second, although the entire sample was randomly split into two samples to maximize the comparability (i.e., age, gender, and teaching age), the biological variables of the two samples may still differ. The questionnaire was insufficient sample and the sample capacity did not cover all regions and major cities across the country. Therefore, research conclusions cannot accurately infer the difference of demographic variables (Liu, 2018). At the same time, research is undertaken in the context of cross-cultural research. All participants are from China, which belongs to an Asian context, and cannot represent all countries and cultures. Future research can be conducted in a wider scope (e.g., Global South) to obtain more comprehensive data (Liu et al. 2020). The generalization of conclusions has yet to be tested through more investigations. These interesting issues are worth further examination.

\section{Data availability}

The data is not publicly available, please contact the corresponding author, if necessary.

Received: 8 November 2019; Accepted: 14 February 2020;

Published online: 10 March 2020

\section{References}

Bagozzi RP, Yi Y (1988) On the evaluation of structural equation models. Academic Mark Sci 16:76-94
Bondebjerg I (2017) The creative mind: cognition, society and culture. Pal Commun $3: 1-7$

Burton JP, Holtom BC, Sablynski CJ et al. (2010) The buffering effects of job embeddedness on negative shocks. J Vocational Behav 76:42-51

Clark SC (2000) Work/family border theory: a new theory of work/family balance. Hum Relat 53(6):747-770

Cohen J, Cohen P, West SG et al. (2013) Applied multiple regression/correlation analysis for the behavioral sciences. Routledge, New York

Crossley CD, Bennett RJ, Jex SM et al. (2007) Development of a global measure of job embeddedness and Integration into a traditional model of voluntary turnover. J Appl Psychol 92:1031-1042

Edwaeds JR, Cable DM, Williamson IO et al. (2006) The phenomenology of fit: linking the person and environment to the subjective experience of personenvironment fit. J Appl Psychol 91(4):802-827

Hair JF, Black WC, Babin BJ et al. (2010) Multivariate data analysis: a global perspective. Prentice-Hall, Upper Sadder River

Kasier HF (1974) Little Jiffy, Mark IV. Educ Psychological Meas 34(1):111-117

Kline RB (2011) Principles and practice of structural equation modeling, 3rd edn. Guilford Press, New York

Lee TW, Sablynski CJ, Burton JP et al. (2004) The effects of job embeddedness on organizational citizenship, job performance, volitional absences, and voluntary turnover. Acad Manag J 47(1):711-722

Liu R (2018) A study on the job embeddedness of the mid-high-end talents. Beijing institute of technology press, Beijing

Liu Z, Lei SH, Guo YL et al. (2020) The interaction effect of online review language style and product type on consumers' purchase intentions. Pal Commun 6:1-8

Mallol CM, Holtom BC, Lee TW (2007) Job embeddedness in a culturally diverse environment. J Bus Psychol 22(1):35-44

McClelland DC (1961) The achieving society. Van Nostrand Reinhold, New York

Meyers LS, Gamst G, Guarino AJ (2017) Applied multivariate research: design and interpretation, 3rd edn. SAGE, Thousand Oaks

Mitchell TR, Holtom BC, Lee TW et al. (2001) Why people stay: using job embeddedness to predict voluntary turnover. Acad Manag J 44(6):1102-1121

Rajappan S, Nair RS, Priyadarshini MK et al. (2017) Exploring the effect of workplace spirituality on job embeddedness among higher secondary school teachers in Ernakulam district. Cogent Bus Manag 4:1-9

Lev. S, Koslowsky M (2012) Teacher gender as a moderator of the on-the-job embeddedness-OCB relationship. J Appl Soc Psychol 42(1):81-99

Song CQ (2016) Modification of the foreign "job embeddedness" model in the context of China. J Guangdong Polytech Norm Univ 4:103-111

Wu ML (2013) Questionnaire statistical analysis practice-SPSS operation and application. Chongqing University Press, Chongqing

Wu ML (2013) Structural equation model Amos practice advanced. Chongqing University Press, Chongqing

Yang CJ, Liu W, Luo B et al. (2013) What should employees think before leaving: job embeddedness in China. Sci-Technol Manag 15(1):25-29

Young-bohk C, Jeong-ran R (2009) Organizational citizenship behaviors in relation to job embeddedness, organizational Identification, job performance, voluntary turnover intention In Korea. Int Bus Econ Res J 24(3):15-20

Zhang ZS (2002) Work-family relations theory and work-family balance program. Nankai Bus Rev 4:55-59

Zhang CN (2011) Empical Study on The High School Teacher's Job Embeddedness and Its Factors. Master's thesis

Zhang YL, Lu GZ (2017) Preliminary preparation of the high school students studies of envy. Ment Health Educ Prim Secondary Sch 7:7-11

Zhong KN (2015) Employees' Family Embeddedness and Intention to Quit in Chinese Context-The Localization Research of Job Embeddedness. Master's thesis

\section{Acknowledgements}

Thanks to Professor Huang Ailing for her help in data collection and revising it critically for important intellectual content. We also thank some postgraduates for their valuable 
comments on the questionnaire and three psychology teachers for strict supervision during the questionnaire collection process.

\section{Competing interests}

The authors declare no competing interests.

\section{Additional information}

Correspondence and requests for materials should be addressed to X.S.

Reprints and permission information is available at http://www.nature.com/reprints

Publisher's note Springer Nature remains neutral with regard to jurisdictional claims in published maps and institutional affiliations. (c) (i) Open Access This article is licensed under a Creative Commons Attribution 4.0 International License, which permits use, sharing, adaptation, distribution and reproduction in any medium or format, as long as you give appropriate credit to the original author(s) and the source, provide a link to the Creative Commons license, and indicate if changes were made. The images or other third party material in this article are included in the article's Creative Commons license, unless indicated otherwise in a credit line to the material. If material is not included in the article's Creative Commons license and your intended use is not permitted by statutory regulation or exceeds the permitted use, you will need to obtain permission directly from the copyright holder. To view a copy of this license, visit http://creativecommons.org/ licenses/by/4.0/.

(C) The Author(s) 2020 